\title{
Current Perspectives on the Systemic Management of Atopic Dermatitis
}

\author{
Danielle R Davari (D) \\ Elizabeth L Nieman (D) \\ Diana B McShane \\ Dean S Morrell
}

Department of Dermatology, University of North Carolina School of Medicine,

Chapel Hill, NC, USA
Correspondence: Dean S Morrell Department of Dermatology, University of North Carolina School of Medicine,

Chapel Hill, NC, USA

Tel +I 9849743900

Fax + I 9849743692

Email morrell@med.unc.edu

\begin{abstract}
Atopic dermatitis (AD) is a common disease of childhood, and some patients experience a prolonged clinical course into adolescence and adulthood. Systemic management is required when $\mathrm{AD}$ is not adequately controlled with topical medications. Our aim is to provide a comprehensive review of commonly used systemic immunomodulating agents in childhood and adult $\mathrm{AD}$, including cyclosporine $\mathrm{A}(\mathrm{Cs} \mathrm{A})$, azathioprine (AZA), methotrexate (MTX) and mycophenolate mofetil (MMF), which are prescribed off-label in the United States, as well as dupilumab, an FDA-approved biologic. We will also provide a brief overview of emerging systemic therapies currently under investigation.
\end{abstract}

Keywords: eczema, adults, pediatric, treatment, dupilumab

\section{Introduction}

Atopic dermatitis (AD) is an inflammatory skin disorder affecting up to $20 \%$ of children and $10 \%$ of adults worldwide. ${ }^{1-4}$ In the United States, the estimated annual cost of AD was $\$ 4.228$ billion in 2004, which is the equivalent of approximately $\$ 5.972$ billion in 2021. 5 The amount of out-of-pocket costs for patients with AD is high, and this burden increases with disease severity. ${ }^{7}$ Quality of life is significantly impacted in patients and their families, and is also correlated with disease severity. ${ }^{8}$ Diagnosis of $\mathrm{AD}$ is made based on clinical signs and symptoms, and the lack of uniform diagnostic criteria and availability of an objective test to confirm diagnosis impacts prevalence estimates. The Hanifin and Rajka criteria is the most widely used diagnostic criteria, followed by the United Kingdom Working Party criteria. ${ }^{9-11}$ Common clinical features of AD include pruritus, xerosis, lichenification, flexural involvement, disease course influenced by emotional and/or environmental factors, and early onset of disease. ${ }^{12}$ Numerous measures for AD severity have been established, such as the Eczema Area and Severity Index (EASI) and the Severity Scoring of Atopic Dermatitis index (SCORAD), which are the best validated and used to assess the clinical severity of $\mathrm{AD} .{ }^{13}$ For the EASI and SCORAD clinical signs are typically assessed by a physician, while the PatientOriented Eczema Measure (POEM) is a preferred tool for patient-reported outcomes. $^{14}$

The three main phases of AD are infantile, childhood, and adult. Since the use of systemic agents in infantile AD is rarely advised, the focus of this review will be on the childhood and adult phases. ${ }^{15}$ The infantile phase may manifest from birth to 2 years of age, followed by the childhood phase from 2 years through puberty. The adult phase begins at puberty and may persist throughout adulthood. ${ }^{16} \mathrm{AD}$ is part of 
the "atopic march", which is an association and potential progression of allergic conditions that develop during infancy and childhood when allergen exposure through inflamed skin may trigger an immunologic response. While not all atopic individuals progress through all features, the atopic march classically begins with $\mathrm{AD}$, followed by IgE-mediated food allergies, asthma and allergic rhinitis. ${ }^{17}$

Childhood AD exhibits lichenified papules and plaques on the hands, feet, wrists and ankles, as well as the antecubital and popliteal regions. Localization at flexural areas is common, although extensor surfaces may be primarily involved in some patients. When there is facial involvement, it tends to affect the periorbital and perioral areas. ${ }^{16}$ In African American children, the lesions are often more papular and severe compared to white children, with structural racism contributing to this disparity. ${ }^{16,18}$ Adult $\mathrm{AD}$ is characterized by dry scaling erythematous papules and plaques, as well as the formation of large lichenified plaques representing chronic disease. The affected areas include the flexural folds, face and neck, upper arms and back, and dorsa of the hands, fingers, feet and toes. ${ }^{16}$ Persistence of AD into adulthood is common and is particularly prevalent in those with early onset, allergic rhinitis, and hand eczema in childhood. ${ }^{19}$

\section{Pathogenesis}

The pathogenesis of $\mathrm{AD}$ is multifactorial and involves a strong genetic component, dysfunctional epidermal barrier and type-2-dominated cutaneous inflammation. ${ }^{20}$ Loss-of-function genetic variants in the gene encoding filaggrin $(F L G)$ predispose individuals to AD. Filaggrin is a protein that facilitates formation of the skin barrier, preventing epidermal water loss and impeding the entry of allergens, toxic chemicals, and infectious organisms. ${ }^{21}$ Barrier dysfunction may also be caused by mechanical damage, such as repetitive scratching, and alterations in the skin microbiome that lead to increased Staphylococcus aureus colonization and susceptibility to cutaneous infections. ${ }^{22,23}$ Cutaneous inflammation in AD is skewed towards $\mathrm{TH} 2$ cell-mediated pathways, with activated $\mathrm{TH} 2$ cells releasing IL-4 and IL-13, which promotes B cell IgE class switching and production of antigen-specific IgE. ${ }^{24}$ The goal of AD management is to control inflammation, improve symptoms and achieve long-term disease control. While most patients with $\mathrm{AD}$ are effectively managed with topical medications, others may require systemic therapy. Our aim is to provide a comprehensive review of commonly used systemic immunomodulating agents in childhood and adult $\mathrm{AD}$, which broadly target cutaneous inflammation, as well as a brief overview of emerging systemic therapies that target specific aspects of $\mathrm{AD}$ pathogenesis.

\section{Literature Search}

A systematic search of the PubMed database was performed to identify contributions associated with systemic treatment of childhood and adult AD. Searches were limited to publications in the English language. MeSH terms used in various combinations in the literature search included: atopic dermatitis, eczema, treatment, systemic therapy, emerging therapy, cyclosporine A, azathioprine, methotrexate, mycophenolate mofetil, dupilumab, tralokinumab, lebrikizumab, nemolizumab, fezakinumab, baricitinib, upadacitinib, and abrocitinib. ClinicalTrials.gov was explored using the terms atopic dermatitis, dupilumab, tralokinumab, lebrikizumab, nemolizumab, fezakinumab, baricitinib, upadacitinib, and abrocitinib.

\section{Systemic Therapy}

Systemic therapy is required to control skin inflammation, reduce symptoms, prevent flares, and improve quality of life in children and adults with moderate to severe AD that does not respond to maximized topical therapies. ${ }^{25}$ A consensus statement from the International Eczema Council recommends that prior to prescribing systemic therapy, physicians should consider alternate or concomitant diagnoses, avoid triggering factors, optimize topical therapy, ensure adequate patient/caregiver education, treat coexistent infection, assess the impact on quality of life, and consider phototherapy. ${ }^{25}$ Commonly used conventional systemic immunomodulating agents include cyclosporine A (CsA), azathioprine (AZA), methotrexate (MTX), and mycophenolate mofetil (MMF), which are prescribed off-label in the US. Dupilumab is an FDAapproved biologic indicated for the treatment of moderate to severe $\mathrm{AD}$ in patients aged 6 years and older. ${ }^{26}$ Many additional biologic and small-molecule medications, such as oral Janus kinase (JAK) inhibitors, are being investigated in clinical trials. We will not discuss systemic corticosteroids, since they are not recommended and their use should be reserved for acute, severe exacerbations as a short-term bridge to another systemic, steroid-sparing agent. ${ }^{27}$ Systemic antibiotics and antiviral agents are recommended in patients with bacterial infections and eczema herpeticum, respectively. ${ }^{27}$ 
Systematic reviews and meta-analyses comparing systemic therapies have shown that CsA and dupilumab may have superior short-term effectiveness compared to MTX and AZA for the treatment of adult AD. ${ }^{28,29}$ A survey completed by dermatologists from 30 European countries revealed that CsA was the preferred first-line conventional systemic therapy, while AZA and MTX were selected as second-line treatments. Conventional systemic therapy options offered in the survey were CsA, AZA, MTX, MMF and oral corticosteroids, while dupilumab was not included. ${ }^{30}$ In 2014, prior to the approval of dupilumab, another survey completed by members of the Society for Pediatric Dermatology in the US and Canada showed that the most commonly chosen first-line agents were CsA and MTX, second-line agents were MTX and MMF, and thirdline agents were AZA and MMF. ${ }^{31}$ With the exception of dupilumab, systemic immunomodulating agents do not have formal dosing guidelines for $\mathrm{AD}$ and require laboratory monitoring (Table 1). Regardless of general prescribing practices, the choice of systemic therapy should be based on each individual patient's needs. Ultimately, physicians should engage in shared decision-making with their patients, taking into account efficacy and safety, as well as convenience and cost when prescribing systemic therapies.

\section{Cyclosporine A (CsA)}

CsA is a potent immunosuppressive agent that inhibits calcineurin via a complex formed between CsA and cyclophilin. Calcineurin inhibition leads to reduced transcription of IL-2, which results in a decline in the number of activated CD4+ and CD8+ T-cells in the epidermis. ${ }^{32}$ It is indicated for the prevention of organ rejection in kidney, liver, and heart allogeneic transplants. It is also indicated for the treatment of patients with rheumatoid arthritis that have not adequately responded to MTX and for patients with plaque psoriasis who have failed to respond to at least one systemic therapy. ${ }^{33} \mathrm{CsA}$ is an effective off-label treatment for childhood and adult $\mathrm{AD}$, and it is approved to treat adult $\mathrm{AD}$ in Europe. ${ }^{32}$ A double-blind, placebo-controlled, crossover RCT of 33 adults with severe refractory AD showed significant improvements in disease activity and extent of disease scores with 8 weeks of treatment compared to placebo. Once CsA was stopped, relapse was rapid, but the mean scores for disease activity and extent of disease were less than their baseline values. ${ }^{34}$ Another double-blind, placebo-controlled RCT of 46 adults with severe AD showed significant improvements in disease severity, extent of disease, lichenification, itch, and loss of sleep with 6 weeks of treatment compared to placebo. ${ }^{35}$ An open, uncontrolled study of 43 adults with severe AD studying the length of remission with CsA treatment suggested that while a significant decrease in disease activity was observed after 6 weeks of CsA, most patients relapsed a few weeks after treatment ended. ${ }^{36}$ An open, uncontrolled study of 27 children aged 2 to 16 years with severe refractory $\mathrm{AD}$ demonstrated a significant improvement in disease activity and quality of life with 6 weeks of treatment. Most children relapsed within a few weeks of stopping treatment, however, persistent improvement was observed after 6 months of follow-up in 3 children. ${ }^{37}$ An open RCT of 43 children aged 2 to 16 years with severe refractory $\mathrm{AD}$ comparing multiple short courses of CsA with continuous therapy for 1 year revealed that more consistent $\mathrm{AD}$ control was achieved with continuous treatment. However, short course therapy was adequate for some patients, indicating that treatment should be tailored to individual patient's needs. ${ }^{38} \mathrm{~A}$ case series of 5 children aged 10 to 14 years with severe AD demonstrated that weekend CsA maintenance therapy may be an option in pediatric patients requiring CsA treatment longer than 1 year. Intermittent therapy on Saturdays and Sundays for 20 weeks resulted in a reduction in the cumulative dose of CsA and serum CsA levels without the loss of clinical benefits. ${ }^{39}$ A systematic review of 15 studies including 602 children and adults with AD showed a mean clinical improvement of about $55 \%$ after 6 to 8 weeks of CsA treatment. Studies analyzing the stability of therapy showed that $50 \%$ of patients relapsed within 2 weeks and $80 \%$ within 6 weeks after stopping treatment. ${ }^{40}$

Adverse reactions of CsA include renal dysfunction, headache, hypertension, hypertriglyceridemia, hirsutism/ hypertrichosis, paresthesia or hyperesthesia, influenzalike symptoms, gastrointestinal (GI) disturbances, lethargy, musculoskeletal or joint pain, and gingival hypertrophy. Due to an increased risk of skin and lymphoproliferative malignancies, patients should not be treated concurrently with CsA and phototherapy, and photoprotection should be advised. ${ }^{33}$ In the systematic review of patients with $A D$ treated with CsA, higher initial doses led to a more rapid response, however, withdrawals from treatment due to adverse events were more likely in patients treated with higher initial doses. The adverse events included GI symptoms, infections, paresthesia and headaches, as well as increases in creatinine and blood pressure. Interestingly, 


\begin{tabular}{|c|c|c|c|c|c|c|}
\hline 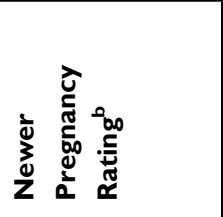 & \multicolumn{2}{|l|}{ 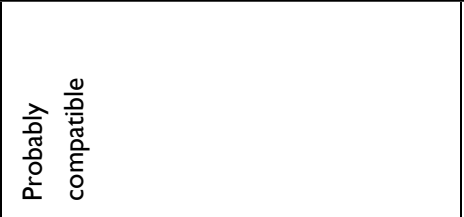 } & \multicolumn{3}{|c|}{ 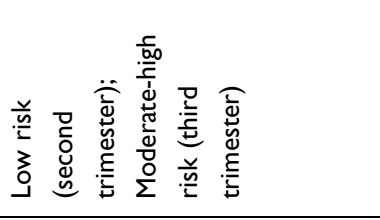 } & 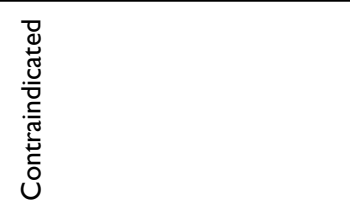 \\
\hline 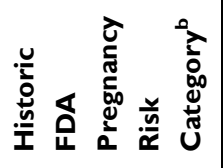 & & \multicolumn{3}{|l|}{$\begin{array}{l}0 \\
\lambda \\
0 \\
0 \\
0 \\
0 \\
0 \\
0\end{array}$} & 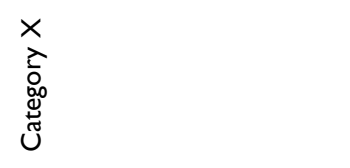 \\
\hline 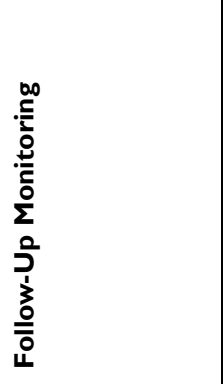 & \multicolumn{2}{|c|}{ 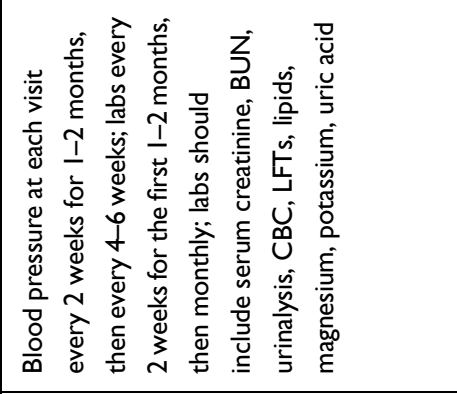 } & \multicolumn{3}{|c|}{ 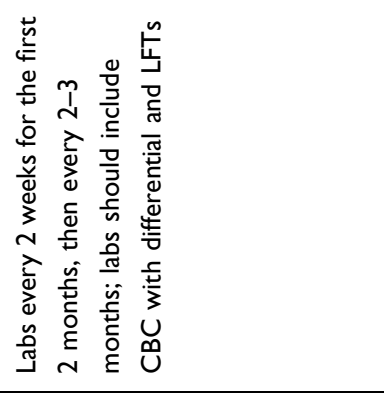 } & 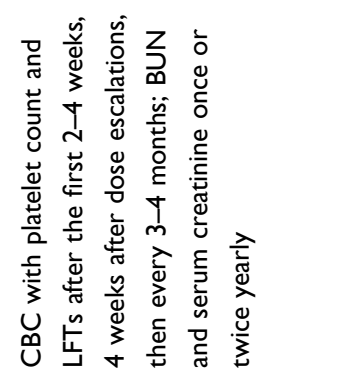 \\
\hline 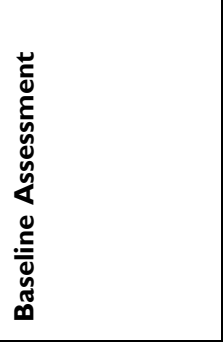 & \multicolumn{2}{|c|}{ 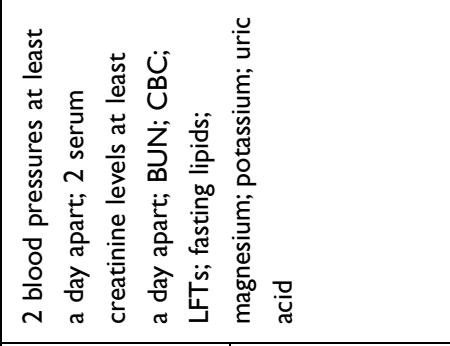 } & \multicolumn{3}{|c|}{ 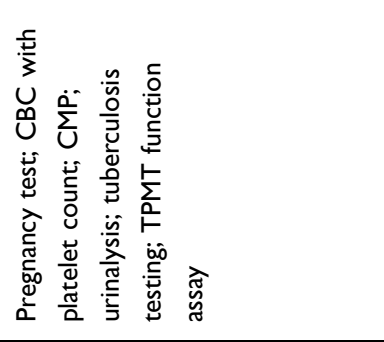 } & 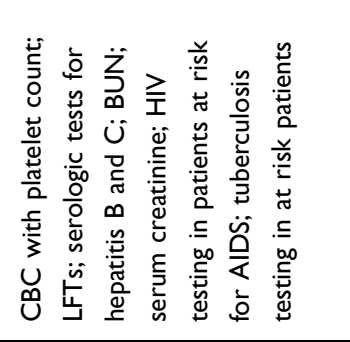 \\
\hline 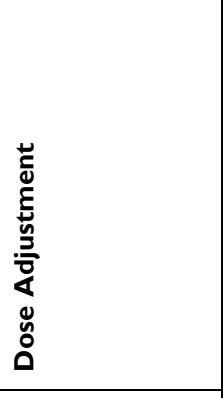 & 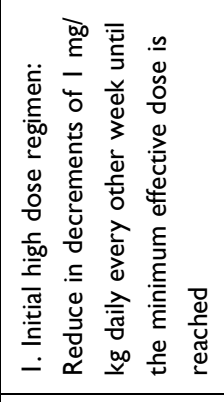 & 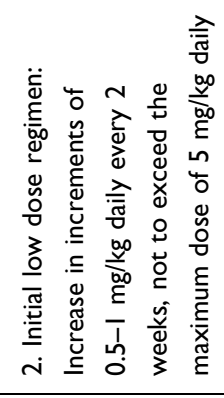 & \multicolumn{3}{|c|}{ 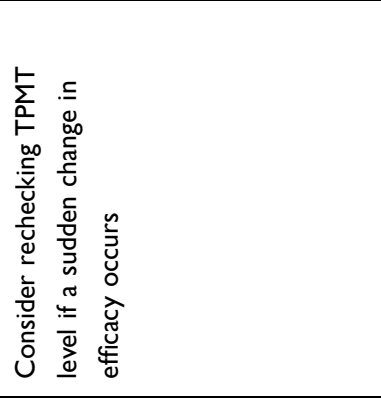 } & 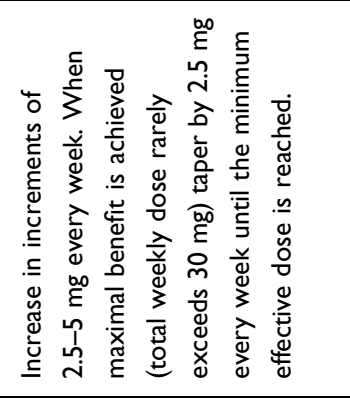 \\
\hline 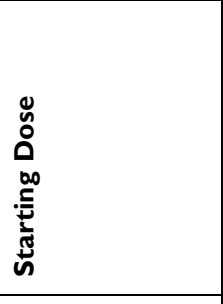 & 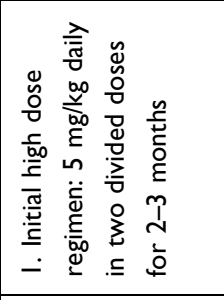 & 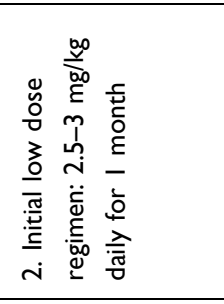 & 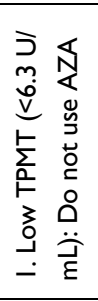 & 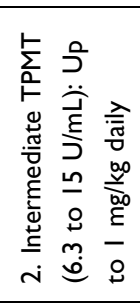 & 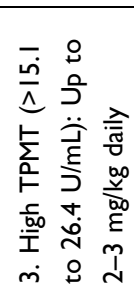 & 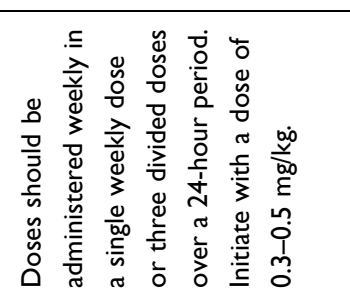 \\
\hline 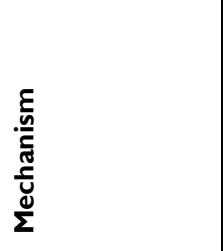 & 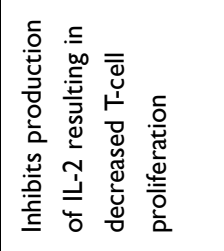 & & 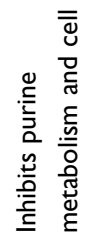 & 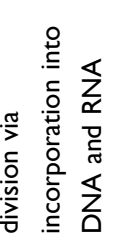 & & 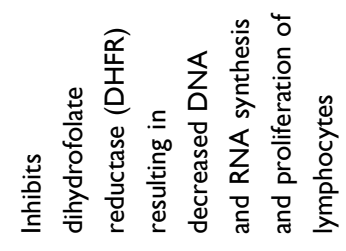 \\
\hline 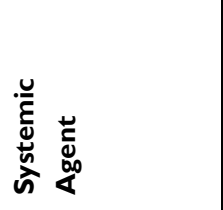 & 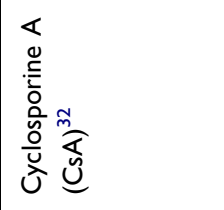 & & 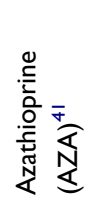 & & & 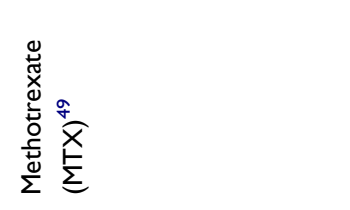 \\
\hline
\end{tabular}


while the effectiveness of CsA in adults and children was similar, tolerability appeared better in children. ${ }^{40}$

There is robust evidence that CsA is effective in the management of children and adults with severe AD. It has a rapid onset of action and an acceptable short-term adverse effect profile. However, relapse is common upon discontinuation of treatment, and potential long-term side effects are concerning.

\section{Azathioprine (AZA)}

AZA is a purine analog that inhibits purine metabolism and cell division when incorporated into DNA and RNA. It also interferes with T-cell, B-cell, and antigen-presenting cell functions. ${ }^{41}$ It is indicated as an adjunct for the prevention of rejection in renal homotransplantations and for the management of active rheumatoid arthritis. ${ }^{42}$ AZA may be used as an off-label treatment for childhood and adult $\mathrm{AD} .{ }^{41} \mathrm{~A}$ double-blind, placebo-controlled, crossover RCT of 37 adults with severe AD showed significant improvements in Six Area, Six Sign Atopic Dermatitis (SASSAD) scores, pruritus, sleep disturbance, and disruption of work and daytime activities during 3 months of treatment compared to placebo. ${ }^{43}$ Another double-blind, placebo-controlled RCT of 63 adults with moderate to severe AD showed significant improvements in SASSAD scores, body area affected, itch score, global response, and quality of life with 3 months of treatment compared to placebo. ${ }^{44}$ In a case series of 28 children aged 3 to 17 years with severe $\mathrm{AD}, 61 \%$ of patients reported significant improvement while being treated with AZA and 21\% reported some improvement. Patients that responded to AZA treatment experienced an initial improvement at a mean duration of 5.3 weeks. ${ }^{45}$

Adverse reactions of AZA include myelosuppression, GI disturbances, and hepatotoxicity. A GI hypersensitivity reaction characterized by nausea and vomiting may develop within the first several weeks of therapy, which is reversible upon discontinuation of the drug. There is an increased risk of malignancy, including post-transplant lymphoma and hepatosplenic T-cell lymphoma, which have been reported in patients with inflammatory bowel disease. Photoprotection should be advised due to increased risk for skin cancer. ${ }^{42}$ AZA is metabolized via three competing pathways, and is either anabolized to its active form by hypoxanthine-guanine phosphoribosyltransferase (HGPRT), or degraded to inactive metabolites by thiopurine methyltransferase (TPMT) or xanthine oxidase (XO). TPMT activity is controlled by a genetic polymorphism, and patients can have high activity, intermediate activity, or low activity. ${ }^{41}$ Patients with intermediate TPMT activity may be at increased risk of myelotoxicity, while those with low or absent TPMT activity are at increased risk of developing severe, lifethreatening myelotoxicity. Therefore, TPMT genotyping or phenotyping (red blood cell TPMT activity) is recommended to identify patients who are at increased risk of toxicity. ${ }^{42}$ In the RCT of 63 adults with moderate to severe AD, TPMT-based dosing seemed to reduce toxicity while maintaining efficacy. ${ }^{44}$ A retrospective study of 91 children aged 6 to 16 years with severe AD showed that AZA had an acceptable short-term adverse effect profile in those with normal TPMT activity. ${ }^{46}$ Case reports demonstrated that children with severe $\mathrm{AD}$ and intermediate TPMT activity responded to treatment with AZA at half-standard doses without any adverse events. ${ }^{47}$ A prospective study of 12 children aged 2 to 18 years with severe, recalcitrant AD revealed that TPMT activity may change during AZA treatment, suggesting that repeated measurement may be useful to optimize dosing. ${ }^{48}$

AZA has been shown to be effective in the management of children and adults with severe AD. It has a favorable safety profile when TPMT function testing is conducted prior to treatment to guide dosing.

\section{Methotrexate (MTX)}

MTX is a folic acid antagonist that inhibits dihydrofolate reductase (DHFR), preventing the conversion of dihydrofolate to tetrahydrofolate. This interferes with DNA and RNA synthesis, as well as the proliferation of lymphocytes. ${ }^{49}$ It is indicated in the treatment of neoplastic diseases such as gestational choriocarcinoma, breast cancer, lung cancer, and non-Hodgkin lymphomas. It is also indicated in the symptomatic control of psoriasis that is not responsive to other forms of therapy, active rheumatoid arthritis, and polyarticular-course juvenile rheumatoid arthritis that is not responsive to first-line therapy. ${ }^{50}$ MTX may be used as an off-label treatment for recalcitrant childhood and adult $\mathrm{AD} .{ }^{49}$ An open-label, dose-ranging, prospective trial of 12 adults with moderate to severe $A D$ treated with MTX for 24 weeks demonstrated an improvement in disease activity of $52 \%$ from baseline, with the majority of improvement observed by week $12 .{ }^{51}$ A single-blind RCT of 42 adults with severe AD comparing treatment with MTX versus AZA and showed a mean SCORAD reduction of $42 \%$ in the MTX arm and 
a reduction of $39 \%$ in the AZA arm after 12 weeks. ${ }^{52}$ To assess the long-term efficacy and safety of MTX and AZA, the 42 patients were asked to participate in an open-label follow-up study for 5 years, which demonstrated that both MTX and AZA seem to be effective and safe as maintenance treatments. ${ }^{53,54}$ An RCT of 97 adults with moderate to severe AD comparing treatment with MTX versus CsA demonstrated that while MTX was less efficient than CsA at lower doses, when the doses of both drugs were increased this led to an EASI reduction of $92 \%$ in the MTX arm versus a reduction of $87 \%$ in the CsA arm. ${ }^{55}$ An RCT of 40 children aged 7 to 14 years with severe AD comparing treatment with MTX versus CsA showed a mean SCORAD reduction of $26 \%$ in the MTX arm and a reduction of $25 \%$ in the CsA arm after 12 weeks. MTX had a delayed onset of action compared to CsA, however, the efficacy of MTX was prolonged. ${ }^{56}$

Common adverse reactions of MTX include ulcerative stomatitis, leukopenia, GI disturbances, malaise, chills and fever, dizziness and decreased resistance to infection. Serious adverse reactions include myelosuppression, hepatotoxicity, methotrexate-induced lung disease, and acute renal failure. Certain side effects may be reduced by folate supplementation. ${ }^{50}$ The RCT of adults with severe AD comparing treatment with MTX versus AZA and showed that mild myelosuppression was more frequent in the AZA arm, with otherwise similar rates of non-severe adverse events. ${ }^{52}$ The RCT of adults with moderate to severe AD comparing treatment with MTX versus CsA revealed that the number of adverse events was significantly higher in the CsA arm (55\%) compared to the MTX arm $(30 \%) .{ }^{55}$ The RCT of children with severe AD comparing treatment with MTX versus CsA showed that the safety profile of both drugs were similar. Common side effects in the MTX arm included anemia, fatigue, abnormal liver functions, nausea and vomiting, and glossitis with oral ulceration. All adverse events resolved at the end of a 12 week follow-up period. ${ }^{56}$

While MTX has demonstrated similar efficacy to CsA and AZA in the management of childhood and adult AD, it has not been studied as extensively. CsA led to a more rapid clinical improvement in children, however, the effects of MTX were longer-lasting. MTX is generally well tolerated, and in adults, the number of adverse events with MTX may be lower compared to CsA.

\section{Mycophenolate Mofetil (MMF)}

MMF is an immunosuppressant that inhibits inosine monophosphate dehydrogenase (IMPDH), a key enzyme in the de novo pathway of purine synthesis. This inhibition leads to reduced lymphocyte proliferation. ${ }^{57}$ It is indicated for the prophylaxis of organ rejection, in recipients of allogeneic kidney, heart or liver transplants. ${ }^{58}$ MMF may be used as an off-label treatment for recalcitrant childhood and adult AD. ${ }^{57}$ A single-blind RCT of 55 adults with severe $\mathrm{AD}$ comparing treatment with $\mathrm{MMF}$ versus CsA showed that while the initial clinical response to MMF was delayed compared to that of CsA, clinical remission lasted longer for patients treated with MMF during a 12 week follow-up period. ${ }^{59}$ An open-label pilot study of 10 adults with moderate to severe AD showed that 7 patients had cleared completely after 4 weeks of MMF treatment and 6 patients had lasting remission at the end of a 20 week follow-up period. ${ }^{60}$ In another open-label pilot study, 10 adults with severe AD achieved a mean SCORAD improvement of $68 \%$ with MMF therapy. ${ }^{61}$ A systematic review of 18 studies including 140 children and adults with AD showed a significant reduction in SCORAD scores with MMF treatment. The average time for initial effects to be observed was 6.8 weeks, and relapses occurred in $8.2 \%$ of cases. ${ }^{62}$

Common adverse reactions of MMF include leukopenia, infection, GI disturbances and decreased resistance to infection. Serious adverse reactions include neutropenia and pure red cell aplasia. There is an increased risk of developing lymphomas and other malignancies, particularly of the skin, and photoprotection should be advised ${ }^{58}$ In the systematic review of patients with $\mathrm{AD}$ treated with MMF, the majority of adverse effects were transient or mild. The most common side effects were headaches and GI discomfort. Anemia, leukopenia, neutropenia and thrombocytopenia were rare. Herpes infection occurred in $9.3 \%$ of patients, which was significantly associated with a prolonged treatment duration. ${ }^{62}$ Although viral and bacterial infections have been described in patients undergoing treatment with MMF, a patient with a history of recurrent eczema herpeticum actually experienced a decrease in frequency and severity of viral outbreaks in a retrospective case series of 14 children aged 2 to 16 years with refractory $\mathrm{AD}$ treated with MMF. ${ }^{63}$ In another retrospective case series of 28 children with severe AD aged 3 to 17 years previously mentioned in the AZA section, 12 of the 28 patients transitioned from AZA to MMF due to medication intolerance and/or failure to respond to AZA. Two-thirds of the patients reported significant improvement with MMF, and 
experienced seemingly less-severe side effects compared to AZA. ${ }^{45}$

MMF has been shown to be effective in the management of adults and children with severe $\mathrm{AD}$, however, the evidence is rather limited. It has an acceptable short-term adverse effect profile and may be better tolerated compared to AZA.

\section{Dupilumab}

Dupilumab is a human monoclonal antibody that directly binds the IL-4 receptor $\alpha$-subunit, which blocks downstream signaling of IL-4 and IL-13. ${ }^{64}$ It is indicated for the treatment of moderate to severe AD in patients aged 6 years and older whose disease is not adequately controlled with topical prescription therapies or when those therapies are not advisable. It is also indicated as a maintenance treatment for certain patients with moderate to severe asthma and for patients with inadequately controlled chronic rhinosinusitis with nasal polyposis. ${ }^{26}$ In earlyphase, double-blind, placebo-controlled RCTs of adults with moderate to severe $\mathrm{AD}$, patients treated with dupilumab improved in all evaluated measures of $\mathrm{AD}$ disease activity. ${ }^{65}$ Two randomized, placebo-controlled, phase 3 trials of 1379 adults with moderate to severe AD showed that 16 weeks of dupilumab treatment was superior to placebo in improving the signs and symptoms of $\mathrm{AD}$, including pruritus, symptoms of anxiety and depression, and quality of life. ${ }^{66}$ A randomized, double-blind, placebocontrolled, phase 3 trial of 740 adults with moderate to severe AD revealed that adding dupilumab to topical corticosteroids (TCS) improved efficacy across multiple measures of clinical severity compared to placebo plus TCS over 52 weeks. ${ }^{67}$ In a randomized, double-blind, placebocontrolled, phase 3 trial of 251 children aged 12 to 17 years with moderate to severe $\mathrm{AD}$, dupilumab significantly improved AD signs, symptoms, and quality of life compared to placebo after 12 weeks. ${ }^{68}$ In an ongoing phase 3 open-label extension study of pediatric patients, which continues to enroll by invitation, 36 children aged 12 to 17 years treated with dupilumab for 52 weeks experienced sustained improvements in disease severity. ${ }^{69,70}$ A randomized, double-blind, placebo-controlled, phase 3 trial of 367 children aged 6 to 11 years with severe AD demonstrated that patients treated with dupilumab plus TCS for 16 weeks had a significant improvement in Investigator's Global Assessment (IGA) compared to those treated with placebo plus TCS. $^{71}$
Common adverse reactions of dupilumab include injection site reactions, conjunctivitis, blepharitis, oral herpes, keratitis, eye pruritus, other herpes complex virus infections, and dry eye. ${ }^{26}$ An analysis of three randomized, double-blind, placebo-controlled phase 3 trials of 2116 adults with moderate to severe AD revealed that conjunctivitis and injection site reactions were more frequent in patients treated with dupilumab compared to placebo. A small number of transient reductions in neutrophils and platelets were observed, as well as transient increases in eosinophils. These changes in laboratory parameters were not associated with any clinical adverse events. This supports the current guidance that there is no requirement for routine laboratory monitoring before initiation or during treatment with dupilumab. ${ }^{72}$ The open-label extension study of pediatric patients found that 52 weeks of dupilumab treatment had a comparable safety profile to 16 weeks of treatment, supporting the long-term use of dupilumab in adolescents. ${ }^{69}$ The trial of children aged 6 to 11 years showed that dupilumab was well tolerated and the most frequently reported side effects were upper respiratory tract infections, nasopharyngitis, headache, vomiting, injection site erythema and conjunctivitis. ${ }^{71}$ Notably, in most AD trials, patients treated with dupilumab had a higher incidence of conjunctivitis compared to placebo, which was associated with higher baseline AD severity and a prior history of conjunctivitis. Most cases were mild to moderate and resolved while continuing dupilumab treatment. $^{73}$

Currently, dupilumab is the only FDA-approved biologic treatment for childhood and adult $\mathrm{AD}$. It has been shown to be efficacious in numerous clinical trials, and despite reports of injection site reactions and conjunctivitis, it has an overall favorable safety profile. Dupilumab is considered the first-line systemic therapy of choice in the management of $\mathrm{AD}$ given its effectiveness upon clinical manifestations, excellent benefit/risk profile, and positive impact upon patients' and families' quality of life. While dupilumab is not indicated to treat infantile $\mathrm{AD}$, a phase 2/ 3 study investigating the pharmacokinetics, safety, and efficacy of dupilumab in patients aged 6 months to 5 years with moderate to severe $\mathrm{AD}$ is currently recruiting. ${ }^{74}$

\section{Emerging Systemic Therapies}

Emerging systemic therapies for AD include biologics and oral JAK inhibitors. Tralokinumab and lebrikizumab are monoclonal antibodies that target IL-13, nemolizumab targets IL-31, and fezakinumab targets IL-22. ${ }^{64}$ Phase 2 
and 3 trials have demonstrated that tralokinumab is an effective and well tolerated treatment option for adults with moderate to severe AD. ${ }^{75-77}$ An ongoing phase 3 trial is evaluating the efficacy of tralokinumab compared to placebo in treating adolescents aged 12 to 17 years with moderate to severe AD. ${ }^{78}$ A long-term extension phase 3 trial enrolling by invitation is evaluating the safety of tralokinumab in patients aged 12 years and older with $\mathrm{AD} .^{79}$ Phase 2 trials have shown that lebrikizumab is efficacious with a favorable safety profile in adults with moderate to severe AD. ${ }^{80,81}$ Phase 3 trials are recruiting to assess the efficacy and safety of lebrikizumab in patients aged 12 years and older with moderate to severe AD. ${ }^{82-86}$ Phase 2 studies have demonstrated that nemolizumab results in rapid and sustained improvements and is well tolerated in adults with moderate to severe AD. ${ }^{87-89}$ A phase 3 trial of patients aged 13 years and older with $\mathrm{AD}$ and moderate to severe pruritus showed that nemolizumab reduced pruritus. ${ }^{90}$ A phase 2 study assessing the pharmacokinetics and safety of nemolizumab in adolescents aged 12 to 17 years with moderate to severe $\mathrm{AD}$ has been completed, however, the results have not yet been posted. ${ }^{91}$ Phase 3 studies are recruiting to evaluate the safety and efficacy of nemolizumab in patients aged 12 years and older with moderate to severe AD. ${ }^{92-94}$ A phase 2 trial showed that fezakinumab had a favorable safety profile in adults with moderate to severe AD, with significant clinical improvements primarily observed in severe AD patients. ${ }^{95}$

Oral JAK inhibitors currently being investigated include baricitinib, upadacitinib, and abrocitinib, which target cytokines involved in the pathogenesis of AD. ${ }^{96}$ In phase 2 and 3 trials, baricitinib significantly improved the signs and symptoms of adults with moderate to severe $\mathrm{AD}$ with an acceptable safety profile. ${ }^{97-101}$ Phase 3 studies are recruiting and ongoing to evaluate the safety and efficacy of baricitinib in patients aged 2 years and older with AD. ${ }^{102,103}$ A phase 2 trial demonstrated that upadacitinib was efficacious with a favorable benefit/risk profile in adults with moderate to severe AD. ${ }^{104}$ Phase 3 trials are recruiting and ongoing to assess the efficacy and safety of upadacitinib in patients aged 12 years and older with moderate to severe AD. ${ }^{105-109}$ A phase 1 study is recruiting to evaluate the safety, pharmacokinetics and tolerability of upadacitinib in pediatric patients aged 6 months to 12 years with severe AD. ${ }^{110}$ Phase 2 and 3 trials showed that abrocitinib was effective and well tolerated in patients aged 12 years and older with moderate to severe AD. ${ }^{111-114}$
A phase 3 study is enrolling by invitation to evaluate the efficacy and safety of abrocitinib in patients aged 12 years and older with moderate to severe $\mathrm{AD}$ over variable treatment periods. ${ }^{115}$ Overall, the results of clinical trials exploring the efficacy and safety of these emerging therapies have been promising. Continued investigations are necessary to evaluate long-term efficacy and safety, especially in children, and such studies are ongoing.

\section{Conclusion}

Systemic therapy may be warranted in childhood and adult $\mathrm{AD}$ that is not adequately controlled with topical medications. Conventional systemic immunomodulating agents are commonly used off-label, with CsA demonstrating the greatest short-term efficacy. However, for patients requiring more long-term treatment the side effects of CsA are concerning, thus, alternative therapies such as AZA, MTX, and MMF should be considered. Dupilumab is currently the only biologic approved as a first-line treatment for moderate to severe $\mathrm{AD}$ in patients aged 6 years and older, and has changed the landscape of $\mathrm{AD}$ treatment. Additional biologic and small-molecule medications targeting specific inflammatory pathways have shown promising results in clinical trials and these studies are ongoing. Ultimately, the choice of systemic therapy depends on each individual patient's needs, and patient education is essential to improve treatment adherence.

\section{Disclosure}

Dr. Nieman was a principal investigator in an AbbVie trial for upadacitinib (M16-049 trial) and a sub-investigator in a Pfizer trial for a JAK inhibitor (JADE 1036 trial). Dr. McShane was a sub-investigator in a now closed Regeneron investigational study for dupilumab. The authors report no other potential conflicts of interest for this work.

\section{References}

1. Odhiambo JA, Williams HC, Clayton TO, Robertson CF, Asher MI. Global variations in prevalence of eczema symptoms in children from ISAAC Phase Three. J Allergy Clin Immunol. 2009;124(6):1251-8. e23. doi:10.1016/j.jaci.2009.10.009

2. Garg N, Silverberg JI. Epidemiology of childhood atopic dermatitis. Clin Dermatol. 2015;33(3):281-288. doi:10.1016/j. clindermatol.2014.12.004

3. Silverberg JI, Hanifin JM. Adult eczema prevalence and associations with asthma and other health and demographic factors: a US population-based study. $J$ Allergy Clin Immunol. 2013;132 (5):1132-1138. doi:10.1016/j.jaci.2013.08.031 
4. Silverberg JI. Public health burden and epidemiology of atopic dermatitis. Dermatol Clin. 2017;35(3):283-289. doi:10.1016/j. $\operatorname{det} .2017 .02 .002$

5. Bickers DR, Lim HW, Margolis D, et al. The burden of skin diseases: 2004 a joint project of the American Academy of Dermatology Association and the Society for Investigative Dermatology. J Am Acad Dermatol. 2006;55(3):490-500. doi:10.1016/j.jaad.2006.05.048

6. CPI Inflation Calculator. U.S. Bureau of labor statistics. Available from: https://www.bls.gov/data/inflation_calculator. htm. Accessed February 12, 2021.

7. Launois R, Ezzedine K, Cabout E, et al. Importance of out-ofpocket costs for adult patients with atopic dermatitis in France. J Eur Acad Dermatol Venereol. 2019;33(10):1921-1927. doi: $10.1111 /$ jdv. 15581

8. Koszorú K, Borza J, Gulácsi L, Sárdy M. Quality of life in patients with atopic dermatitis. Cutis. 2019;104(3):174-177.

9. Hanifin J, Rajka G. Diagnostic features of atopic-dermatitis. Acta Derm Venereol. 1980;92:44-47.

10. Williams HC, Burney PG, Hay RJ, et al. The U.K. Working Party's Diagnostic Criteria for atopic dermatitis. I. Derivation of a minimum set of discriminators for atopic dermatitis. $\mathrm{Br}$ $J \quad$ Dermatol. 1994;131(3):383-396. doi:10.1111/j.13652133.1994.tb08530.x

11. Vakharia PP, Chopra R, Silverberg JI. Systematic review of diagnostic criteria used in atopic dermatitis randomized controlled trials. Am J Clin Dermatol. 2018;19(1):15-22. doi:10.1007/ s40257-017-0299-4

12. Yew YW, Thyssen JP, Silverberg JI. A systematic review and meta-analysis of the regional and age-related differences in atopic dermatitis clinical characteristics. $J$ Am Acad Dermatol. 2019;80 (2):390-401. doi:10.1016/j.jaad.2018.09.035

13. Schmitt J, Langan S, Deckert S, et al. Assessment of clinical signs of atopic dermatitis: a systematic review and recommendation. J Allergy Clin Immunol. 2013;132(6):1337-1347. doi:10.1016/j. jaci.2013.07.008

14. Vakharia PP, Cella D, Silverberg JI. Patient-reported outcomes and quality of life measures in atopic dermatitis. Clin Dermatol. 2018;36(5):616-630. doi:10.1016/j.clindermatol. 2018.05.011

15. Davari DR, Nieman EL, McShane DB, Morrell DS. Current perspectives on the management of infantile atopic dermatitis. $J$ Asthma Allergy. 2020;13:563-573. doi:10.2147/jaa.S246175

16. Spergel JM, Paller AS. Atopic dermatitis and the atopic march. J Allergy Clin Immunol. 2003;112(6Suppl):S118-27. doi:10.1016/ j.jaci.2003.09.033

17. Hill DA, Spergel JM. The atopic march: critical evidence and clinical relevance. Ann Allergy Asthma Immunol. 2018;120 (2):131-137. doi:10.1016/j.anai.2017.10.037

18. Tackett KJ, Jenkins F, Morrell DS, McShane DB, Burkhart CN. Structural racism and its influence on the severity of atopic dermatitis in African American children. Pediatr Dermatol. 2020;37(1):142-146. doi:10.1111/pde.14058

19. Mortz CG, Andersen KE, Dellgren C, Barington T, BindslevJensen C. Atopic dermatitis from adolescence to adulthood in the TOACS cohort: prevalence, persistence and comorbidities. Allergy. 2015;70(7):836-845. doi:10.1111/all.12619

20. Langan SM, Irvine AD, Weidinger S. Atopic dermatitis. Lancet. 2020;396(10247):345-360. doi:10.1016/s0140-6736(20)31286-1

21. Palmer CN, Irvine AD, Terron-Kwiatkowski A, et al. Common loss-of-function variants of the epidermal barrier protein filaggrin are a major predisposing factor for atopic dermatitis. Nat Genet. 2006;38(4):441-446. doi:10.1038/ng1767

22. Tsakok T, Woolf R, Smith CH, Weidinger S, Flohr C. Atopic dermatitis: the skin barrier and beyond. Br J Dermatol. 2019;180 (3):464-474. doi:10.1111/bjd.16934
23. Powers $\mathrm{CE}$, McShane DB, Gilligan PH, Burkhart CN, Morrell DS. Microbiome and pediatric atopic dermatitis. $J$ Dermatol. 2015;42(12):1137-1142. doi:10.1111/13468138.13072

24. Weidinger S, Beck LA, Bieber T, Kabashima K, Irvine AD. Atopic dermatitis. Nat Rev Dis Primers. 2018;4(1):1. doi:10.1038/s41572-018-0001-z

25. Simpson EL, Bruin-Weller M, Flohr C, et al. When does atopic dermatitis warrant systemic therapy? Recommendations from an expert panel of the International Eczema Council. $J$ Am Acad Dermatol. 2017;77(4):623-633. doi:10.1016/j.jaad.2017.06.042

26. Dupixent ${ }^{\circledR}$ (Dupilumab) Injection [Prescribing Information]. Tarrytown: Regeneron Pharmaceuticals, Inc; 2020.

27. Sidbury R, Davis DM, Cohen DE, et al. Guidelines of care for the management of atopic dermatitis: section 3. Management and treatment with phototherapy and systemic agents. $\mathrm{J} \mathrm{Am} \mathrm{Acad}$ Dermatol. 2014;71(2):327-349. doi:10.1016/j.jaad.2014.03.030

28. Seger EW, Wechter T, Strowd L, Feldman SR. Relative efficacy of systemic treatments for atopic dermatitis. $J$ Am Acad Dermatol. 2019;80(2):411-416.e4. doi:10.1016/j.jaad.2018.09.053

29. Drucker AM, Ellis AG, Bohdanowicz M, et al. Systemic immunomodulatory treatments for patients with atopic dermatitis: a systematic review and network meta-analysis. JAMA Dermatol. 2020;156(6):659-667. doi:10.1001/jamadermatol.2020.0796

30. Vermeulen FM, Gerbens LAA, Schmitt J, et al. The European TREatment of ATopic eczema (TREAT) Registry Taskforce survey: prescribing practices in Europe for phototherapy and systemic therapy in adult patients with moderate-to-severe atopic eczema. Br J Dermatol. 2020;183(6):1073-1082. doi:10.1111/ bjd. 18959

31. Totri CR, Eichenfield LF, Logan K, et al. Prescribing practices for systemic agents in the treatment of severe pediatric atopic dermatitis in the US and Canada: the PeDRA TREAT survey. $\mathrm{J} \mathrm{Am} \mathrm{Acad}$ Dermatol. 2017;76(2):281-285. doi:10.1016/j.jaad.2016.09.021

32. Sekhon S, Bhutani T, Koo JYM. 17 - Cyclosporine. In: Wolverton SE, editor. Comprehensive Dermatologic Drug Therapy (Fourth Edition). Elsevier; 2021:187-198.e3.

33. Neoral ${ }^{\circledR}$ (Cyclosporine) Capsules [Prescribing Information]. East Hanover: Novartis Pharmaceuticals Corporation; 2009.

34. Sowden JM, Berth-Jones J, Ross JS, et al. Double-blind, controlled, crossover study of cyclosporin in adults with severe refractory atopic dermatitis. Lancet. 1991;338(8760):137-140. doi:10.1016/0140-6736(91)90134-b

35. van Joost $\mathrm{T}$, Heule F, Korstanje M, van den Broek MJ, Stenveld HJ, van Vloten WA. Cyclosporin in atopic dermatitis: a multicentre placebo-controlled study. Br J Dermatol. 1994;130 (5):634-640. doi:10.1111/j.1365-2133.1994.tb13111.x

36. Granlund H, Erkko P, Sinisalo M, Reitamo S. Cyclosporin in atopic dermatitis: time to relapse and effect of intermittent therapy. $B r \quad J$ Dermatol. 1995;132(1):106-112. doi:10.1111/ j.1365-2133.1995.tb08633.x

37. Berth-Jones J, Finlay AY, Zaki I, et al. Cyclosporine in severe childhood atopic dermatitis: a multicenter study. J Am Acad Dermatol. 1996;34(6):1016-1021. doi:10.1016/s0190-9622(96)90281-9

38. Harper JI, Ahmed I, Barclay G, et al. Cyclosporin for severe childhood atopic dermatitis: short course versus continuous therapy. Br J Dermatol. 2000;142(1):52-58. doi:10.1046/j.13652133.2000.03241.x

39. Garrido Colmenero C, Blasco Morente G, Tercedor Sánchez J. Oral cyclosporine weekend therapy: a new maintenance therapeutic option in patients with severe atopic dermatitis. Pediatr Dermatol. 2015;32(4):551-552. doi:10.1111/pde.12592

40. Schmitt J, Schmitt N, Meurer M. Cyclosporin in the treatment of patients with atopic eczema - a systematic review and meta-analysis. $J$ Eur Acad Dermatol Venereol. 2007;21 (5):606-619. doi:10.1111/j.1468-3083.2006.02023.x 
41. Grove D, Rahnama-Moghadam S. 15 - Azathioprine. In: Wolverton SE, editor. Comprehensive Dermatologic Drug Therapy (Fourth Edition). Elsevier; 2021:169-177.e3.

42. Imuran ${ }^{\circledR}$ (Azathioprine) Tablets 50mg [Prescribing Information]. San Diego: Prometheus Laboratories Inc; 2011.

43. Berth-Jones J, Takwale A, Tan E, et al. Azathioprine in severe adult atopic dermatitis: a double-blind, placebo-controlled, crossover trial. Br J Dermatol. 2002;147(2):324-330. doi:10.1046/ j.1365-2133.2002.04989.x

44. Meggitt SJ, Gray JC, Reynolds NJ. Azathioprine dosed by thiopurine methyltransferase activity for moderate-to-severe atopic eczema: a double-blind, randomised controlled trial. Lancet. 2006;367(9513):839-846. doi:10.1016/s0140-6736(06)68340-2

45. Waxweiler WT, Agans R, Morrell DS. Systemic treatment of pediatric atopic dermatitis with azathioprine and mycophenolate mofetil. Pediatr Dermatol. 2011;28(6):689-694. doi:10.1111/ j.1525-1470.2011.01488.x

46. Murphy LA, Atherton D. A retrospective evaluation of azathioprine in severe childhood atopic eczema, using thiopurine methyltransferase levels to exclude patients at high risk of myelosuppression. $\mathrm{Br} \quad \mathrm{J}$ Dermatol. 2002;147(2):308-315. doi:10.1046/j.1365-2133.2002.04922.x

47. Murphy LA, Atherton DJ. Azathioprine as a treatment for severe atopic eczema in children with a partial thiopurine methyl transferase (TPMT) deficiency. Pediatr Dermatol. 2003;20 (6):531-534. doi:10.1111/j.1525-1470.2003.20617.x

48. Caufield M, Tom WL. Oral azathioprine for recalcitrant pediatric atopic dermatitis: clinical response and thiopurine monitoring $J$ Am Acad Dermatol. 2013;68(1):29-35. doi:10.1016/j. jaad.2012.07.001

49. Callen JP, Kulp-Shorten CL. 14 - Methotrexate. In: Wolverton SE, editor. Comprehensive Dermatologic Drug Therapy (Fourth Edition). Elsevier; 2021:156-168.e5.

50. Methotrexate Tablets [Prescribing Information]. Huntsville: Qualitest Pharmaceuticals; 2016.

51. Weatherhead SC, Wahie S, Reynolds NJ, Meggitt SJ. An open-label, dose-ranging study of methotrexate for moderate-tosevere adult atopic eczema. Br J Dermatol. 2007;156(2):346-351. doi:10.1111/j.1365-2133.2006.07686.x

52. Schram ME, Roekevisch E, Leeflang MM, Bos JD, Schmitt J, Spuls PI. A randomized trial of methotrexate versus azathioprine for severe atopic eczema. J Allergy Clin Immunol. 2011;128 (2):353-359. doi:10.1016/j.jaci.2011.03.024

53. Roekevisch E, Schram ME, Leeflang MMG, et al. Methotrexate versus azathioprine in patients with atopic dermatitis: 2-year follow-up data. $J$ Allergy Clin Immunol. 2018;141(2):825-827. e10. doi:10.1016/j.jaci.2017.09.033

54. Gerbens LAA, Hamann SAS, Brouwer MWD, Roekevisch E, Leeflang MMG, Spuls PI. Methotrexate and azathioprine for severe atopic dermatitis: a 5-year follow-up study of a randomized controlled trial. $\mathrm{Br} J$ Dermatol. 2018;178 (6):1288-1296. doi:10.1111/bjd.16240

55. Goujon C, Viguier M, Staumont-Sallé D, et al. Methotrexate versus cyclosporine in adults with moderate-to-severe atopic dermatitis: a Phase III Randomized Noninferiority Trial. J Allergy Clin Immunol Pract. 2018;6(2):562-569.e3. doi:10.1016/j. jaip.2017.07.007

56. El-Khalawany MA, Hassan H, Shaaban D, Ghonaim N, Eassa B. Methotrexate versus cyclosporine in the treatment of severe atopic dermatitis in children: a multicenter experience from Egypt. Eur J Pediatr. 2013;172(3):351-356. doi:10.1007/s00431-0121893-3

57. Braue JA, Schadt CR, Zwerner JP. 16 - Mycophenolates. In: Wolverton SE, editor. Comprehensive Dermatologic Drug Therapy (Fourth Edition). Elsevier; 2021:178-186.e4.
58. Cellcept ${ }^{(\mathbb{R}}$ (Mycophenolate Mofetil) Capsules [Prescribing Information]. South San Francisco: Genentech, Inc; 2018.

59. Haeck IM, Knol MJ, Ten Berge O, van Velsen SG, De bruinweller MS, Bruijnzeel-Koomen CA. Enteric-coated mycophenolate sodium versus cyclosporin A as long-term treatment in adult patients with severe atopic dermatitis: a randomized controlled trial. J Am Acad Dermatol. 2011;64(6):1074-1084. doi:10.1016/j. jaad.2010.04.027

60. Grundmann-Kollmann M, Podda M, Ochsendorf F, Boehncke WH, Kaufmann R, Zollner TM. Mycophenolate mofetil is effective in the treatment of atopic dermatitis. Arch Dermatol. 2001;137(7):870-873.

61. Neuber K, Schwartz I, Itschert G, Dieck AT. Treatment of atopic eczema with oral mycophenolate mofetil. $\mathrm{Br} J$ Dermatol. 2000;143(2):385-391. doi:10.1046/j.1365-2133.2000.03667.x

62. Phan K, Smith SD. Mycophenolate mofetil and atopic dermatitis: systematic review and meta-analysis. $J$ Dermatolog Treat. 2020;31(8):810-814. doi:10.1080/09546634.2019.1642996

63. Heller M, Shin HT, Orlow SJ, Schaffer JV. Mycophenolate mofetil for severe childhood atopic dermatitis: experience in 14 patients. Br J Dermatol. 2007;157(1):127-132. doi:10.1111/ j.1365-2133.2007.07947.x

64. Grinich EE, Simpson EL. 31 - Additional biologic therapeutics: dupilumab, omalizumab, others. In: Wolverton SE, editor. Comprehensive Dermatologic Drug Therapy (Fourth Edition). Elsevier; 2021:339-348.e4.

65. Beck LA, Thaçi D, Hamilton JD, et al. Dupilumab treatment in adults with moderate-to-severe atopic dermatitis. $N$ Engl $J$ Med. 2014;371(2):130-139. doi:10.1056/NEJMoa1314768

66. Simpson EL, Bieber T, Guttman-Yassky E, et al. Two Phase 3 Trials of dupilumab versus placebo in atopic dermatitis. $N$ Engl $J$ Med. 2016;375(24):2335-2348. doi:10.1056/NEJMoa1610020

67. Blauvelt A, de Bruin-weller M, Gooderham M, et al. Long-term management of moderate-to-severe atopic dermatitis with dupilumab and concomitant topical corticosteroids (LIBERTY AD CHRONOS): a 1-year, randomised, double-blinded, placebo-controlled, phase 3 trial. Lancet. 2017;389 (10086):2287-2303. doi:10.1016/s0140-6736(17)31191-1

68. Simpson EL, Paller AS, Siegfried EC, et al. Efficacy and safety of dupilumab in adolescents with uncontrolled moderate to severe atopic dermatitis: a Phase 3 Randomized Clinical Trial. JAMA Dermatol. 2020;156(1):44-56. doi:10.1001/jamadermatol.2019.3336

69. Cork MJ, Thaçi D, Eichenfield LF, et al. Dupilumab in adolescents with uncontrolled moderate-to-severe atopic dermatitis: results from a phase IIa open-label trial and subsequent phase III open-label extension. $B r J$ Dermatol. 2020;182(1):85-96. doi:10.1111/bjd.18476

70. Regeneron Pharmaceuticals. Study to assess the long-term safety of dupilumab administered in participants $\geq 6$ months to $<18$ years of age with atopic dermatitis (AD). NLM identifier: NCT02612454. Available from: https://clinicaltrials.gov/ct2/ show/NCT02612454. Accessed February 12, 2021.

71. Regeneron Pharmaceuticals. Study to investigate the efficacy and safety of dupilumab administered with Topical Corticosteroids (TCS) in participants $\geq 6$ to $<12$ years with severe atopic dermatitis (AD). NLM identifier: NCT03345914. Available from: https:/clinicaltrials.gov/ct2/show/NCT03345914?term= NCT03345914\&draw=2\&rank=1. Accessed February 12, 2021.

72. Wollenberg A, Beck LA, Blauvelt A, et al. Laboratory safety of dupilumab in moderate-to-severe atopic dermatitis: results from three phase III trials (LIBERTY AD SOLO 1, LIBERTY AD SOLO 2, LIBERTY AD CHRONOS). Br J Dermatol. 2020;182 (5):1120-1135. doi:10.1111/bjd.18434

73. Akinlade B, Guttman-Yassky E, de Bruin-weller M, et al. Conjunctivitis in dupilumab clinical trials. $\mathrm{Br} J$ Dermatol. 2019;181(3):459-473. doi:10.1111/bjd.17869 
74. Regeneron Pharmaceuticals. Safety, pharmacokinetics and efficacy of dupilumab in patients $\geq 6$ months to $<6$ years with moderate-to-severe atopic dermatitis (liberty AD PRESCHOOL) (Liberty AD). NLM identifier: NCT03346434. Available from: https://clinicaltrials.gov/ct2/show/NCT03346434?term= NCT03346434\&draw=2\&rank=1. Accessed February 12, 2021.

75. Wollenberg A, Howell MD, Guttman-Yassky E, et al. Treatment of atopic dermatitis with tralokinumab, an anti-IL-13 mAb. J Allergy Clin Immunol. 2019;143(1):135-141. doi:10.1016/j.jaci.2018.05.029

76. Wollenberg A, Blauvelt A, Guttman-Yassky E, et al. Tralokinumab for moderate-to-severe atopic dermatitis: results from two 52-week, randomized, double-blind, multicentre, placebo-controlled phase III trials (ECZTRA 1 and ECZTRA 2). Br J Dermatol. 2020. doi:10.1111/bjd.19574

77. Silverberg JI, Toth D, Bieber T, et al. Tralokinumab plus topical corticosteroids for the treatment of moderate-to-severe atopic dermatitis: results from the double-blind, randomized, multicentre, placebo-controlled phase III ECZTRA 3 trial. Br J Dermatol. 2020. doi:10.1111/bjd.19573

78. LEO Pharma. Tralokinumab monotherapy for adolescent subjects with moderate to severe atopic dermatitis - ECZTRA 6 (ECZema TRAlokinumab Trial no. 6). NLM identifier: NCT03526861. Available from: https://clinicaltrials.gov/ct2/show/ NCT03526861?term=NCT03526861\&draw=2\&rank=1. Accessed February 12, 2021.

79. LEO Pharma. Long-term extension Trial in subjects with atopic dermatitis who participated in previous tralokinumab trials ECZTEND. NLM identifier: NCT03587805. Available from: https://clinicaltrials.gov/ct2/show/NCT03587805?term= NCT03587805\&draw=2\&rank=1. Accessed February 12, 2021.

80. Guttman-Yassky E, Blauvelt A, Eichenfield LF, et al. Efficacy and safety of lebrikizumab, a high-affinity interleukin 13 inhibitor, in adults with moderate to severe atopic dermatitis: a Phase $2 \mathrm{~b}$ Randomized Clinical Trial. JAMA Dermatol. 2020;156 (4):411-420. doi:10.1001/jamadermatol.2020.0079

81. Simpson EL, Flohr C, Eichenfield LF, et al. Efficacy and safety of lebrikizumab (an anti-IL-13 monoclonal antibody) in adults with moderate-to-severe atopic dermatitis inadequately controlled by topical corticosteroids: a randomized, placebo-controlled Phase II trial (TREBLE). J Am Acad Dermatol. 2018;78(5):863-871.e11. doi:10.1016/j.jaad.2018.01.017

82. Eli Lilly and Company. Long-term safety and efficacy Study of Lebrikizumab (LY3650150) in participants with moderate-tosevere atopic dermatitis. Available from: NLM identifier: NCT 04392154 . https://clinicaltrials.gov/ct2/show/ NCT04392154?term=NCT04392154\&draw=2\&rank=1.

Accessed February 12, 2021.

83. Eli Lilly and Company. Study to assess the safety and efficacy of lebrikizumab (LY3650150) in adolescent participants with moderate-to-severe atopic dermatitis (ADore). NLM identifier: NCT04250350. Available from: https://clinicaltrials.gov/ct2/show/ NCT04250350?term $=$ NCT04250350\&draw $=2 \&$ rank $=1$. Accessed February 12, 2021.

84. Eli Lilly and Company. Evaluation of the efficacy and safety of Lebrikizumab (LY3650150) in moderate to severe atopic dermatitis (ADvocate2). NLM identifier: NCT04178967. Available from: https://clinicaltrials.gov/ct2/show/NCT04178967?term= NCT04178967\&draw=2\&rank=1. Accessed February 12, 2021.

85. Eli Lilly and Company. Evaluation of the efficacy and safety of Lebrikizumab (LY3650150) in moderate to severe atopic dermatitis (ADvocate1). NLM identifier: NCT04146363. Available from: https://clinicaltrials.gov/ct2/show/NCT04146363?term= NCT04146363\&draw=2\&rank=1. Accessed February 12, 2021.
86. Eli Lilly and Company. Safety and efficacy of Lebrikizumab (LY3650150) in combination with topical corticosteroid in moderate-to-severe atopic dermatitis. (ADhere). NLM identifier: NCT04250337. Available from: https://clinicaltrials.gov/ct2/ show/NCT04250337?term=NCT04250337\&draw=2\&rank=1. Accessed February 12, 2021.

87. Ruzicka T, Hanifin JM, Furue M, et al. Anti-interleukin-31 receptor A antibody for atopic dermatitis. $N$ Engl J Med. 2017;376 (9):826-835. doi:10.1056/NEJMoa1606490

88. Kabashima K, Furue M, Hanifin JM, et al. Nemolizumab in patients with moderate-to-severe atopic dermatitis: randomized, Phase II, long-term extension study. J Allergy Clin Immunol. 2018;142(4):1121-1130.e7. doi:10.1016/j.jaci.2018.03.018

89. Silverberg JI, Pinter A, Pulka G, et al. Phase 2B randomized study of nemolizumab in adults with moderate-to-severe atopic dermatitis and severe pruritus. J Allergy Clin Immunol. 2020;145 (1):173-182. doi:10.1016/j.jaci.2019.08.013

90. Kabashima K, Matsumura T, Komazaki H, Kawashima M. Trial of nemolizumab and topical agents for atopic dermatitis with pruritus. NEngl JMed. 2020;383(2):141-150. doi:10.1056/NEJMoa1917006

91. Galderma R\&D. A pharmacokinetics and safety study of nemolizumab in adolescent subjects with atopic dermatitis (AD). NLM identifier: NCT03921411. Available from: https:/clinicaltrials. gov/ct2/show/NCT03921411?term =NCT03921411\&draw $=$ 2\&rank=1. Accessed February 12, 2021.

92. Galderma R\&D. Long-term safety and efficacy of nemolizumab with moderate-to-severe atopic dermatitis. NLM identifier: NCT03989206. Available from: https://clinicaltrials.gov/ct2/ show/NCT03989206?term=NCT03989206\&draw=2\&rank=1. Accessed February 12, 2021.

93. Galderma R\&D. Efficacy and safety of nemolizumab in subjects with moderate-to-severe atopic dermatitis. NLM identifier: NCT03985943. Available from: https://clinicaltrials.gov/ct2/ show/NCT03985943?term=NCT03985943\&draw=2\&rank=1. Accessed February 12, 2021.

94. Galderma R\&D. Efficacy \& safety of nemolizumab in subjects with moderate-to-severe atopic dermatitis. NLM identifier: NCT03989349. Available from: https://clinicaltrials.gov/ct2/ show/NCT03989349?term=NCT03989349\&draw=2\&rank=1. Accessed February 12, 2021.

95. Guttman-Yassky E, Brunner PM, Neumann AU, et al. Efficacy and safety of fezakinumab (an IL-22 monoclonal antibody) in adults with moderate-to-severe atopic dermatitis inadequately controlled by conventional treatments: a randomized, double-blind, phase 2a trial. J Am Acad Dermatol. 2018;78 (5):872-881.e6. doi:10.1016/j.jaad.2018.01.016

96. Weston G, Strober B. 18 - Phosphodiesterase-4 and Janus Kinase Inhibitors. In: Wolverton SE, editor. Comprehensive Dermatologic Drug Therapy (Fourth Edition). Elsevier; 2021:199-208.e3.

97. Guttman-Yassky E, Silverberg JI, Nemoto O, et al. Baricitinib in adult patients with moderate-to-severe atopic dermatitis: a phase 2 parallel, double-blinded, randomized placebo-controlled multiple-dose study. J Am Acad Dermatol. 2019;80(4):913-921. e9. doi:10.1016/j.jaad.2018.01.018

98. Simpson EL, Lacour JP, Spelman L, et al. Baricitinib in patients with moderate-to-severe atopic dermatitis and inadequate response to topical corticosteroids: results from two randomized monotherapy phase III trials. $B r \quad J$ Dermatol. 2020;183 (2):242-255. doi:10.1111/bjd.18898

99. Reich K, Kabashima K, Peris K, et al. Efficacy and safety of baricitinib combined with topical corticosteroids for treatment of moderate to severe atopic dermatitis: a Randomized Clinical Trial. JAMA Dermatol. 2020;156(12):1333-1343. doi:10.1001/ jamadermatol.2020.3260 
100. Eli Lilly and Company. A Long-term Study of Baricitinib (LY3009104) with topical corticosteroids in adults with moderate to severe atopic dermatitis that are not controlled with cyclosporine or for those who cannot take oral cyclosporine because it is not medically advisable (BREEZE-AD4). NLM identifier: NCT03428100. Available from: https://clinicaltrials.gov/ct2/ show/NCT03428100?term=NCT03428100\&draw $=2 \&$ rank $=1$. Accessed February 12, 2021.

101. Eli Lilly and Company. A Study of Baricitinib (LY3009104) in adult participants with moderate to severe atopic dermatitis (BREEZE-AD5). NLM identifier: NCT03435081. Available from: https:/clinicaltrials.gov/ct2/show/NCT03435081?term= NCT03435081\&draw=2\&rank=1. Accessed February 12, 2021.

102. Eli Lilly and Company. A Study of Baricitinib (LY3009104) in children and adolescents with atopic dermatitis (BREEZE-ADPEDS). NLM identifier: NCT03952559. Available from: https:// clinicaltrials.gov/ct2/show/NCT03952559?term= NCT03952559\&draw=2\&rank=1. Accessed February 12, 2021.

103. Eli Lilly and Company. A Study of Long-term Baricitinib (LY3009104) therapy in atopic dermatitis. NLM identifier: NCT03334435. Available from: https://clinicaltrials.gov/ct2/ show/NCT03334435?term=NCT03334435\&draw=2\&rank=1. Accessed February 12, 2021.

104. Guttman-Yassky E, Thaçi D, Pangan AL, et al. Upadacitinib in adults with moderate to severe atopic dermatitis: 16-week results from a randomized, placebo-controlled trial. J Allergy Clin Immunol. 2020;145(3):877-884. doi:10.1016/j.jaci.2019.11.025

105. AbbVie. Evaluation of upadacitinib in adolescent and adult patients with moderate to severe atopic dermatitis (Eczema) (Measure Up 1). NLM identifier: NCT03569293. Available from: https://clinicaltrials.gov/ct2/show/NCT03569293?term= NCT03569293\&draw=2\&rank=1. Accessed February 12, 2021.

106. AbbVie. A study to evaluate upadacitinib in combination with topical corticosteroids in adolescent and adult participants with moderate to severe atopic dermatitis (AD Up). NLM identifier: NCT03568318. Available from: https://clinicaltrials.gov/ct2/ show/NCT03568318?term=NCT03568318\&draw=2\&rank=1. Accessed February 12, 2021.

107. AbbVie. A study to evaluate safety of upadacitinib in combination with topical corticosteroids in adolescent and adult participants with moderate to severe atopic dermatitis (Rising Up). NLM identifier: NCT03661138. Available from: https://clinical trials.gov/ct2/show/NCT03661138?term=NCT03661138\&draw= 2\&rank=1. Accessed February 12, 2021.
108. AbbVie. A study to evaluate upadacitinib in adolescent and adult subjects with moderate to severe atopic dermatitis (Measure Up 2). NLM identifier: NCT03607422. Available from: https://clini caltrials.gov/ct $2 / \mathrm{show} / \mathrm{NCT} 03607422$ ? term $=$ NCT03607422\&draw=2\&rank=1. Accessed February 12, 2021.

109. AbbVie. Open-label extension study of upadacitinib in adult participants with moderate to severe atopic dermatitis. NLM identifier: NCT04195698. Available from: https://clinicaltrials. gov/ct2/show/NCT04195698? term $=$ NCT04195698\&draw $=$ 2\&rank=1. Accessed February 12, 2021.

110. AbbVie. A study to evaluate the pharmacokinetics, safety and tolerability of upadacitinib in pediatric participants with severe atopic dermatitis. NLM identifier: NCT03646604. Available from: https://clinicaltrials.gov/ct2/show/NCT03646604?term= NCT03646604\&draw=2\&rank=1. Accessed February 12, 2021.

111. Gooderham MJ, Forman SB, Bissonnette R, et al. Efficacy and safety of oral janus kinase 1 inhibitor abrocitinib for patients with atopic dermatitis: a Phase 2 Randomized Clinical Trial. JAMA Dermatol. 2019;155(12):1371-1379. doi:10.1001/ jamadermatol.2019.2855

112. Simpson EL, Sinclair R, Forman S, et al. Efficacy and safety of abrocitinib in adults and adolescents with moderate-to-severe atopic dermatitis (JADE MONO-1): a multicentre, double-blind, randomised, placebo-controlled, phase 3 trial. Lancet. 2020;396 (10246):255-266. doi:10.1016/s0140-6736(20)30732-7

113. Silverberg JI, Simpson EL, Thyssen JP, et al. Efficacy and safety of abrocitinib in patients with moderate-to-severe atopic dermatitis: a Randomized Clinical Trial. JAMA Dermatol. 2020;156 (8):863-873. doi:10.1001/jamadermatol.2020.1406

114. Eichenfield L, Flohr C, Sidbury R, et al. Efficacy and safety of abrocitinib in adolescent patients with moderate-to-severe atopic dermatitis (AD): results From the Phase 3 JADE TEEN study [abstract]. $J$ Allergy Clin Immunol. 2021;147(2):AB146. doi:10.1016/j.jaci.2020.12.529

115. Pfizer. Study to evaluate efficacy and safety of PF-04965842 with or without topical medications in subjects aged 12 years and older with moderate to severe atopic dermatitis (JADE EXTEND). NLM identifier: NCT03422822. Available from: https:/clinical trials.gov/ct2/show/NCT03422822?term=NCT03422822\&draw $=$ 2\&rank=1. Accessed February 12, 2021.

\section{Publish your work in this journal}

The Journal of Asthma and Allergy is an international, peer-reviewed open-access journal publishing original research, reports, editorials and commentaries on the following topics: Asthma; Pulmonary physiology; Asthma related clinical health; Clinical immunology and the immunological basis of disease; Pharmacological interventions and

Submit your manuscript here: https://www.dovepress.com/journal-of-asthma-and-allergy-journal new therapies. The manuscript management system is completely online and includes a very quick and fair peer-review system, which is all easy to use. Visit http://www.dovepress.com/testimonials.php to read real quotes from published authors. 